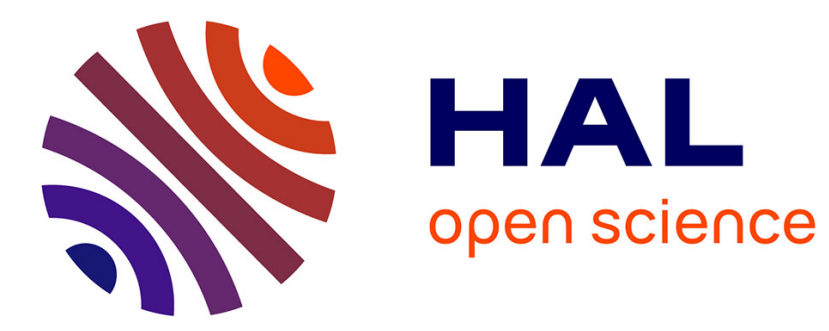

\title{
Instabilités de déformation pendant l'étirage des polymères solides

\author{
C. G'Sell
}

\section{To cite this version:}

C. G'Sell. Instabilités de déformation pendant l'étirage des polymères solides. Revue de Physique Appliquée, 1988, 23 (6), pp.1085-1101. 10.1051/rphysap:019880023060108500 . jpa-00245911

\section{HAL Id: jpa-00245911 https://hal.science/jpa-00245911}

Submitted on 1 Jan 1988

HAL is a multi-disciplinary open access archive for the deposit and dissemination of scientific research documents, whether they are published or not. The documents may come from teaching and research institutions in France or abroad, or from public or private research centers.
L'archive ouverte pluridisciplinaire HAL, est destinée au dépôt et à la diffusion de documents scientifiques de niveau recherche, publiés ou non, émanant des établissements d'enseignement et de recherche français ou étrangers, des laboratoires publics ou privés. 
Classification

Physics Abstracts

$62.20 \mathrm{~F}-46.30 \mathrm{~L}-61.40 \mathrm{~K}$

\title{
Instabilités de déformation pendant l'étirage des polymères solides
}

\author{
C. G’Sell \\ Laboratoire de Physique du Solide, U.A. CNRS 155, Ecole Nationale Supérieure des Mines de Nancy, Parc de \\ Saurupt, 54042 Nancy Cedex, France
}

(Reçu le 30 novembre 1987, accepté le 8 mars 1988)

Article de mise au point

\begin{abstract}
Résumé. - Les procédés de mise en forme des polymères à l'état solide (thermoformage, emboutissage, étirage de films et de fibres) sont affectés par des phénomènes d'instabilités plastiques. Ces instabilités correspondent à différents mécanismes de localisation de la déformation : a) à l'échelle microscopique (quelques $\mathrm{nm}$ ), cascades de changements conformationnels des macromolécules ; b) à l'échelle mésoscopique (quelques $\mu \mathrm{m}$ ), bandes de cisaillement, hétérogénéités intra-sphérolitiques et microcraquelures ; c) à l'échelle macroscopique (quelques $\mathrm{mm}$ ), strictions diffuses et pliages. Ces phénomènes sont interprétés sur la base de la loi de comportement intrinsèque du matériau qui exprime l'influence de la déformation et de la vitesse de déformation sur la contrainte d'écoulement plastique. Cette loi reflète directement les modifications structurales subies par le matériau dans les conditions de l'essai. Par ailleurs, elle donne les informations nécessaires à la modélisation des instabilités à l'aide de techniques de calcul telles que la Méthode des Différences Finies. Deux cas modèles sont étudiés avec une attention particulière : la traction uniaxiale de cylindres de Polyéthylène à Haute Densité d'une part, et l'étirage en déformations planes de films minces de Polytérephtalate d'Ethylène Glycol d'autre part.
\end{abstract}

\begin{abstract}
The forming processes of solid polymers (thermoforming, stamping, film and fiber stretching) are affected by plastic instability phenomena. These instabilities correspond to different strain localization mechanisms : a) at the microscopic scale (nm scale), cascades of conformational changes in macromolecules ; b) at the mesoscopic scale ( $\mu \mathrm{m}$ scale), shear bands, intra-spherulitic inhomogeneities and crazes ; c) at the macroscopic scale (mm scale), diffuse necking and kinking. These phenomena are interpreted in terms of the intrinsic constitutive equation of the material which expresses the influence of strain and strain rate on plastic flow stress. This equation reflects the structural modifications undergone by the material during the deformation. Furthermore, it gives the necessary information for the prediction of strain instabilities by means of computer modelling such as the Finite Difference Method. Two model cases are investigated with a particular attention : the uniaxial tension of High Density Polyethylene cylinders on one hand, and the plane strain stretching of Polyethylene Terephtalate thin films on the other hand.
\end{abstract}

\section{Introduction.}

Dans les processus de mise en forme des matériaux à l'état solide, les phénomènes d'instabilité plastique contrôlent souvent l'aspect et les performances du produit fini. L'étude de ces phénomènes revêt donc une grande importance scientifique et technologique. Dans les procédés généralement utilisés pour les métaux (emboutissage, forgeage, tréfilage, laminage), la formation de bandes de cisaillement et de strictions diffuses a été largement discutée $[1,2]$ et fait encore l'objet de nombreuses publications. Dans le cas des matériaux polymères, le problème des instabilités de déformation a été longtemps délaissé car la plupart des objets manufacturés en matière plastique sont issus de procédés de mise en forme à l'état fondu (moulage par injection, extrusion, calandrage...) dont l'étude relève plutôt d'approches de mécanique des fluides. Cependant, les procédés de mise en forme à basse température se développent où le polymère est le plus souvent déformé à l'état solide : étirage de produits longs, biétirage de films, thermoformage de plaques, forgeage en matrice fermée, extrusion hydrostatique avec ou sans étirage ultérieur, estampage, laminage [3-11]. Comme dans les métaux, des bandes de cisaillement et des stric- 
tions diffuses sont observées et ici encore, leur développement incontrôlé peut être nuisible à la qualité du produit [12]. Toutefois, la cinétique de croissance de ces instabilités est très différente de celles observées dans les métaux : à titre d'exemple, la striction des polymères ductiles apparaît beaucoup plus tôt que dans les métaux mais elle tend à se propager à partir d'une certaine déformation critique au lieu de s'aggraver jusqu'à rupture.

Il est donc indispensable de bien comprendre les mécanismes de l'instabilité plastique dans les polymères afin de savoir les contrôler. Pour cela, il faut établir les relations qui lient la cinétique de développement des gradients de déformation à la loi de comportement plastique intrinsèque du matériau. Le problème est assez complexe quand le matériau est soumis à une déformation triaxiale généralisée comme c'est souvent le cas dans les procédés industriels. Dans le présent travail, nous nous intéressons seulement au cas d'étirages simples. Dans un premier temps, nous rappellerons quelques notions structurales concernant les matériaux polymères. Nous examinerons ensuite les caractères particuliers de leur comportement plastique. Nous analyserons les différents types d'instabilités qui y sont observés et nous développerons enfin les bases théoriques de l'étude des strictions diffuses.

\section{Caractères spécifiques de la structure et des propriétés des polymères solides.}

2.1 RAPPELS SUR LA STRUCTURE DES POLYMÈRES. - Le point commun de tous les grands polymères industriels est d'être constitués de longues chaînes macromoléculaires [13]. Chaque macromolécule est faite de plusieurs dizaines à plusieurs milliers de monomères identiques dont le squelette est le plus souvent à base d'atomes de carbone. Les liaisons atomiques, le long d'une chaîne, sont de type covalent : leur énergie est très élevée $(\approx 100 \mathrm{kcal} / \mathrm{mole})$, de sorte qu'en première approximation on peut considérer qu'elles ne peuvent pas se rompre sous l'effet d'une contrainte mécanique appliquée. Malgré leur solidité, les chaînes polymères peuvent avoir une certaine flexibilité si la température est suffisante. Au niveau des liaisons simples Carbone-Carbone en effet, la molécule peut subir une rotation sur le cône de valence (Fig. 1). La mobilité de ce mécanisme est simplement limitée par des effets d'encombrement stérique (par exemple dans le Polystyrène où des groupements benzéniques latéraux sont attachés périodiquement sur le squelette carboné) ainsi que par les interactions secondaires à longue distance (entre atomes de la même chaîne ou surtout entre chaînes différentes). Les interactions secondaires sont en général de type Van-Der-Waals et leur énergie ( $<1 \mathrm{kcal} / \mathrm{mole}$ ) est très inférieure à celle des liaisons covalentes de la

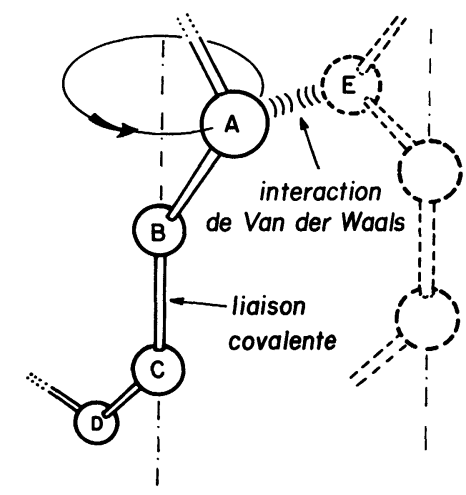

Fig. 1. - Représentation schématique de la rotation d'une chaîne macromoléculaire.

[Schematic diagram of the rotation of a macromolecular chain.]

chaîne. C'est cette dualité des liaisons dans la structure des polymères qui est à l'origine de leurs propriétés mécaniques particulières. Il est utile ici de distinguer les polymères aux molécules indépendantes (thermoplastiques) de ceux dont les chaînes ont été réticulées en établissant entre elles un réseau de liaisons covalentes fortes (thermodurcissables). Bien que les polymères du deuxième type soient largement utilisés pour leur stabilité thermique et leurs bonnes propriétés élastiques (ex. : matrice Epoxide ou Polyester pour matériaux composites), ils ne peuvent pas subir de grands allongements et leur usage est donc impossible dans tous les procédés de transformation où une certaine ductilité est nécessaire. Tous les exemples considérés dans cet article concerneront donc des polymères thermoplastiques à macromolécules chimiquement indépendantes.

Dans un grand nombre de polymères (Polystyrène (PS), Polycarbonate (PC), Polyméthacrylate de Méthyle (PMMA), Polychlorure de vinyle (PVC), Polytérephtalate d'éthylène glycol (PET)...), l'arrangement spatial des macromolécules ne présente aucun ordre à grande distance, ou tout au plus une microcristallinité extrêmement imparfaite comme dans le PVC. Nous considérerons donc en première approximation que ces polymères ont une structure amorphe. Seules, les distances entre atomes proches voisins présentent une certaine régularité (ordre à courte distance). Afin d'augmenter l'entropie de conformation, les chaînes tendent à former des pelotes irrégulières. Les différentes macromolécules sont intimement enchevêtrées les unes dans les autres. Les matériaux qui ont cette structure présentent différents types de comportements en fonction de la température : i) à basse température, le polymère est vitreux, rigide, parfois fragile, avec un module d'Young de l'ordre de $1000 \mathrm{MPa}$, ii) audessus de la température de Transition Vitreuse $T_{\mathrm{g}}$ le comportement mécanique devient viscoélasti- 
que puis caoutchoutique avec un module de l'ordre de $1 \mathrm{MPa}$, le matériau pouvant subir des allongements hyperélastiques considérables [14], iii) à des températures encore plus élevées, l'agitation thermique est telle que la diffusion des chaînes devient très aisée et le comportement est alors celui d'un fluide visqueux (c'est le domaine d'application des procédés de mise en forme à l'état fondu). La figure 2 montre l'évolution typique du module élastique d'un polymère amorphe avec la température.

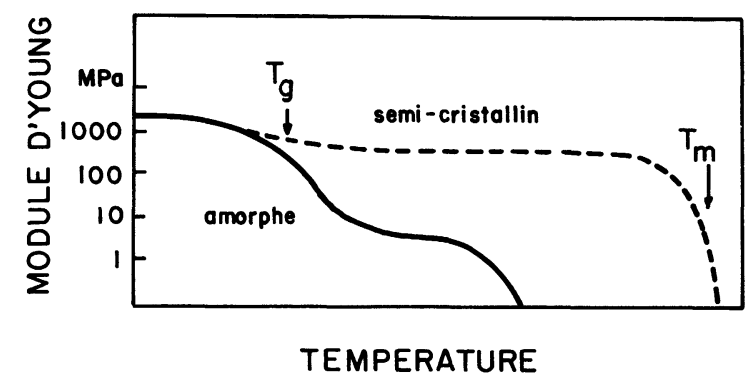

Fig. 2. - Evolution typique du module d'Young des polymères amorphes et semi-cristallins avec la température.

[Typical evolution of Young's Modulus of amorphous and semi-crystalline polymers versus temperature.]

Les polymères dont les chaînes macromoléculaires sont les plus régulières adoptent une structure semicristalline [15]. Pour minimiser l'énergie de conformation, les chaînes se replient sur elles-mêmes pour former des segments réguliers qui s'empilent selon un réseau cristallin de plus forte densité que le polymère amorphe. Cette structure se forme au cours du refroidissement depuis l'état liquide. En raison de la viscosité du polymère fondu et des nombreux défauts des macromolécules, la cristallisation ne peut pas être totale ni parfaite. La structure présente en fin de refroidissement une juxtaposition de cristallites (assez imparfaites) et de zones amorphes résiduelles. Dans les polymères semi-cristallins les plus courants (Polyéthylène -PE-, Polypropylène -PP-, Polyamides -PA-, Polytétra fluoro éthylène PTFE-...), le taux de cristallinité dépasse rarement $70 \%$. Le comportement mécanique de cette classe de matériaux présente donc deux températures caractéristiques: la température de transition vitreuse $T_{\mathrm{g}}$ de la phase amorphe et la température de fusion $T_{\mathrm{m}}$ de la phase cristallisée. On a donc i) en dessous de $T_{\mathrm{g}}$ un polymère rigide de module d'Young voisin des polymères amorphes vitreux, ii) entre $T_{\mathrm{g}}$ et $T_{\mathrm{m}}$ un matériau assez souple (Module d'Young $\approx 700 \mathrm{MPa}$ ) à cause de la phase amorphe caoutchoutique mais avec une bonne résistance grâce à la présence des cristallites et iii) au-dessus de $T_{\mathrm{m}}$ un fluide visqueux. L'évolution du module est également reportée à la figure 2 .

\subsection{MÉCANISMES DE DÉFORMATION DES POLYMÉ-} RES. - Aux températures supérieures à $T_{\mathrm{g}}$, les polymères amorphes caoutchoutiques se déforment de manière progressive, homogène et réversible. Sous l'effet d'une contrainte, les chaînes diffusent rapidement et les pelotes moléculaires s'allongent dans la direction de traction principale. On n'observe pas de seuil d'écoulement. La contrainte augmente régulièrement, mais non linéairement, avec la déformation. La force de retour élastique qui s'oppose à l'extension des chaînes est d'origine entropique, les molécules tendant à revenir à la forme idéale de pelote sphérique qui leur assure une entropie maximum.

Dans le cas des polymères semi-cristallins entre $T_{\mathrm{g}}$ et $T_{\mathrm{m}}$, les petites déformations sont essentiellement assumées par la phase caoutchoutique. Cependant, les cristallites se déforment plastiquement à partir d'une certaine contrainte critique. Comme dans tout cristal ductile, les cristallites polymères subissent un cisaillement plastique localisé dans des plans de glissement par l'effet de mouvements de dislocations. Le durcissement plastique de ces matériaux est dû pour une faible part à la consolidation de cette plasticité cristalline, mais davantage aux effets entropiques de l'orientation moléculaire dans la phase amorphe puis dans les cristallites ellesmêmes qui finissent par se fragmenter en une texture fibreuse fortement anisotrope alignée dans le sens de la traction [16].

Le cas des polymères vitreux est moins bien compris. Aux petites déformations, le comportement est viscoélastique avec une contribution élastique instantanée liée à la distorsion des liaisons de Vander-Waals [17] et une composante retardée due à la relaxation de certaines conformations à haute énergie sous l'effet de réarrangements diffusionnels à courte distance. A plus forte contrainte ces liaisons ne tiennent plus et une déformation irréversible brutale est observée. Dans certains matériaux polymères (PS, PMMA) elle se produit avec une décohésion de la matière par formation de microcraquelures crazes au niveau desquelles les chaînes s'étirent localement entre les lèvres de cavités internes en forme de fissures [18]. Dans les polymères ductiles au contraire (PC, PET, PVC à l'ambiante, et presque tous les matériaux à l'approche de $T_{\mathrm{g}}$ ) la matière se déforme pratiquement sans cavitation. Cette plasticité se produit par cisaillement, les mécanismes qui contrôlent le processus agissant à très courte distance : un petit segment de chaîne emprisonné dans la cage constituée par ses proches voisins subit un basculement qui accommode localement le cisaillement macroscopique [19]. L'activation d'un événement plastique local crée autour du segment concerné un champ de contrainte élastique qui tend à propager le processus au voisinage immédiat du site initial et dans la direction du 
cisaillement macroscopique [20]. Le front de cisaillement ainsi formé a toutes les caractéristiques d'une dislocation par ses propriétés topologiques générales [21-22]. Ce cisaillement reste rarement dispersé dans tout le volume de matière mais se concentre au contraire dans des lignes de glissement d'une fraction de micromètre d'épaisseur [23] car la multiplication des dislocations dans l'état amorphe est un phénomène qui s'entretient spontanément. En d'autres termes, le déclenchement de la plasticité dans un verre polymère est plus difficile que sa propagation car c'est la formation des premiers défauts qui demande la contrainte la plus élevée. Nous verrons plus loin que la formation des lignes de glissement conduit souvent à des instabilités plastiques macrospiques. Aux plus fortes déformations plastiques, les chaînes subissent aussi une orientation progressive dans le sens de l'axe principal des déformations, de sorte que leur distorsion ultérieure devient de plus en plus difficile (durcissement plastique). La rupture survient par des mécanismes d'endommagement.

\subsection{EQUATIONS CONSTITUTIVES DES POLYMÈRES EN TRACTION UNIAXIALE.}

2.3.1 Notion de comportement intrinsèque du matériau. Méthode d'essai de traction à vitesse de déformation vraie constante. - Afin de traiter les instabilités plastiques sur une base quantitative, il est nécessaire de connaître la loi de comportement du matériau, c'est-à-dire l'évolution de la contrainte de traction $\sigma$ avec la déformation $\varepsilon$, et la vitesse de déformation $\dot{\varepsilon}$ à la température $T$. Toutefois, cette loi n'a de sens que si les termes $\sigma, \varepsilon$ et $\dot{\varepsilon}$ peuvent être définis de manière homogène dans un élément de volume donné. Il faut donc les définir à une échelle locale petite par rapport aux dimensions de la striction mais assez grande toutefois pour englober largement les hétérogénéités microscopiques (dislocations, lignes de glissement, sphérolites). Nous avons développé dans ce laboratoire une méthode d'essai de traction originale qui vérifie ces hypothèses et permet de réaliser des essais de traction à vitesse de déformation vraie constante. L'éprouvette est de forme axisymétrique avec un profil en sablier (Fig. 3). A cause de cette symétrie, les strictions diffuses sont favorisées par rapport aux bandes obliques grossières et la localisation se produit toujours au centre. La déformation vraie locale à cet endroit est donnée par la relation

$$
\varepsilon=2 \ln \left(D_{0} / D\right)
$$

où $D_{0}$ et $D$ mesurent le diamètre initial et courant de l'éprouvette. Cette équation suppose vérifiée la constance du volume en cours de déformation plastique, ce qui est assuré en première approximation si la cavitation est négligeable. Le paramètre $\varepsilon$ exprimé par l'équation (1) mesure la déformation totale qui

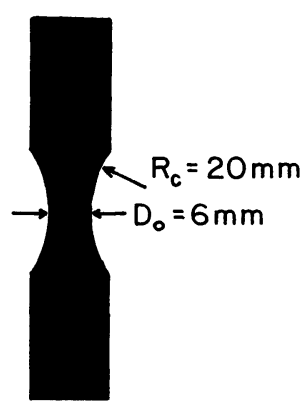

Fig. 3. - Eprouvette en forme de sablier pour essais de traction à vitesse de déformation vraie constante.

[Hourglass shaped specimen for tensile tests at constant true strain rate.]

est la somme des composantes élastique, viscoélastique et plastique. La contrainte vraie locale s'exprime en fonction de la force appliquée $F$ par la relation

$$
\sigma=4 F / \pi D^{2}
$$

Une légère correction de triaxialité est nécessaire si le rayon de courbure $R_{\mathrm{c}}$ du profil devient petit. D'après Bridgman [24], la contrainte uniaxiale effective s'exprime simplement par

$\sigma_{\text {eff }}=\sigma \cdot F_{\mathrm{T}}=\sigma /\left(1+2 R_{\mathrm{c}} / R\right) \times \ln \left(1+R / 2 R_{\mathrm{c}}\right)$

Dans la pratique, le rayon $R=D / 2$ et le rayon de courbure $R_{\mathrm{c}}$ sont mesurés automatiquement par un dispositif vidéo piloté par ordinateur. Afin d'assurer une vitesse de déformation constante $\dot{\varepsilon}$, il est nécessaire d'asservir la traverse de la machine de traction de telle sorte que le diamètre décroisse selon la loi $D=D_{0} \exp (-\dot{\varepsilon} \cdot t / 2)$. Cela est facilité par l'utilisation d'une machine d'essai hydraulique à asservissement électronique [25]. Ce type d'essai est applicable à tous les matériaux ductiles (les polymères, mais aussi les métaux) et à toutes températures.

2.3.2 Comportements typiques des polymères solides. - Les courbes de la figure 4 montrent les différents types de comportement rencontrés dans le cas d'un polymère amorphe, illustrés par les courbes $\sigma(\varepsilon)$ d'une même nuance de PMMA sollicité en traction à différentes températures. A l'ambiante, le verre polymère casse prématurément car le développement des microcraquelures devance la plasticité généralisée. A partir de $70{ }^{\circ} \mathrm{C}$, c'est l'écoulement plastique qui est prépondérant. Un crochet de traction se produit à la fin du stade viscoélastique tandis que se multiplient des microbandes de cisaillement au centre de l'échantillon. La faible pente qui suit le crochet correspond à un faible durcissement structural initial, tandis que le redressement rapide de la courbe en fin d'essai indique l'orientation de plus en plus marquée des chaînes. Au dessus de 


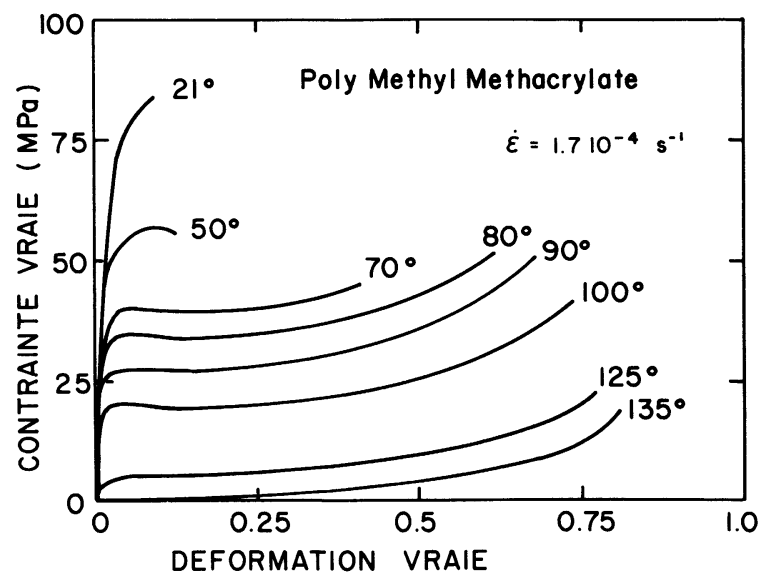

Fig. 4. - Comportement plastique (contrainte vraie/déformation vraie) du Poly (Méthacrylate de Méthyle) amorphe à différentes températures.

[Plastic behaviour (true stress vs. true strain) of amorphous Polymethyl Methacrylate at various temperatures.]

$T_{\mathrm{g}}$, vers $120^{\circ} \mathrm{C}$, le seuil de plasticité disparaît totalement (pas de limite élastique visible) et l'échantillon revient rapidement à l'origine s'il est relaché en cours d'essai : le PMMA est devenu alors un élastomère hyperélastique.

Le Polyéthylène à haute densité - HDPE - de la figure 5 est pour sa part un exemple typique de polymère semi-cristallin. Sa courbe intrinsèque varie aussi avec la température mais sa forme reste pratiquement inchangée depuis les températures cryogéniques (où une transition ductile-fragile apparaît) jusqu'au point de fusion, vers $130^{\circ} \mathrm{C}$, où il passe brutalement à l'état visqueux. On constate que la limite élastique ne présente plus de crochet mais

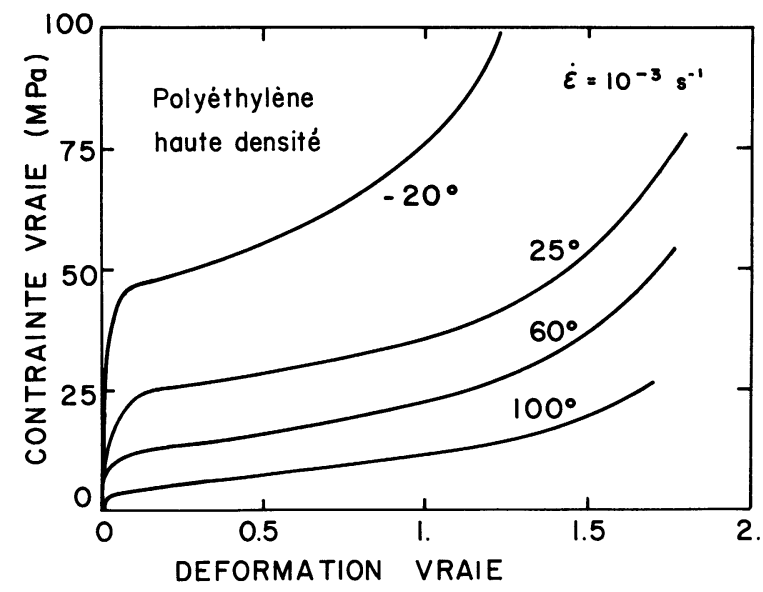

Fig. 5. - Comportement plastique (contrainte vraie/déformation vraie) du Polyéthylène à Haute Densité semicristallin à différentes températures.

[Plastic behaviour (true stress vs. true strain) of High Density Polyethylene at various temperatures.] une transition arrondie progressive. De très fortes déformations sont applicables, jusqu'à des taux d'allongement de l'ordre de $750 \%(\varepsilon=2)$.

Enfin, les courbes de la figure 6 montrent la variété des comportements de divers polymères plastiques ductiles à la température ambiante. Leurs caractères généraux les classent très clairement en différentes catégories :

- Verres amorphes avec

$$
T_{\mathrm{g}}>25^{\circ} \mathrm{C} \quad(\mathrm{PC}, \mathrm{PVC}, \mathrm{PET})
$$

— Elastomères amorphes avec

$$
T_{\mathrm{g}}<25^{\circ} \mathrm{C} \quad \text { (Polyvinyle Butyral PVB) }
$$

- Semi-cristallins avec

$$
T_{\mathrm{g}}<25^{\circ} \mathrm{C}<T_{\mathrm{m}} \quad \text { (PE, PP, PTFE) }
$$

- Semi-cristallins avec

$$
25^{\circ} \mathrm{C}<T_{\mathrm{g}}<T_{\mathrm{m}}
$$

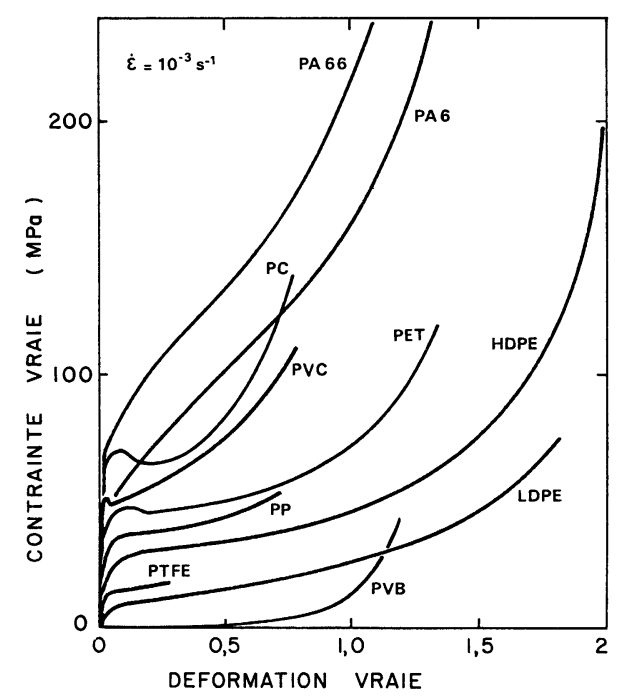

Fig. 6. - Comportement plastique de différents polymères amorphes et semi-cristallins à la température ambiante.

[Plastic behaviour of various amorphous and semi-crystalline polymers at room temperature.]

2.3.3 Définition d'une loi de comportement. - On appelle loi de comportement une relation mathématique qui décrit la dépendance de la contrainte à la déformation et à la vitesse de déformation en traction uniaxiale à une température donnée. Cette loi $\sigma(\varepsilon, \dot{\varepsilon})$ constitue l'équation d'état du matériau dans la mesure où le paramètre de déformation $\varepsilon$ caractérise de manière convenable l'état structural du matériau. Cette propriété n'est vérifiée qu'en première approximation car, pour une valeur fixée de $\varepsilon$, la structure d'un polymère thermoplastique 
peut évoluer par le jeu de phénomènes de relaxation des chaînes (si l'échantillon est soumis à un recuit prolongé à longueur constante par exemple). Cependant, nous avons montré [26] que pour un étirage réalisé dans des conditions normales, cette restriction ne se pose que pendant des périodes transitoires très limitées, par exemple au début du stade plastique (crochet de traction) ou après une période où la vitesse de déformation plastique est brutalement modifiée (saut de vitesse, reprise après maintien prolongé à déformation constante, etc...). D'une manière générale, on peut considérer que $\varepsilon$ est un bon indicateur de l'état structural du matériau car il est lié à la texture d'orientation adoptée par les chaînes pendant la déformation plastique ou hyperélastique. Cela est confirmé par le fait que si une éprouvette est déchargée après étirage, la déformation et la structure tendent à revenir à leur état initial, soit rapidement si la température est supérieure à $T_{\mathrm{g}}$ (ou $T_{\mathrm{m}}$ pour les semi-cristallins), soit très lentement si la température est inférieure. Sous ces hypothèses, nous avons montré que la loi de comportement de tous les polymères étudiés peut s'écrire sous une forme simplifiée unique [25]

$$
\sigma(\varepsilon, \dot{\varepsilon})=K \cdot f(\varepsilon) \cdot g(\dot{\varepsilon})
$$

où $K$ est un facteur d'échelle tandis que $f(\varepsilon)$ et $g(\dot{\varepsilon})$ représentent l'influence de la déformation et de la vitesse de déformation respectivement. Plus spécifiquement, la fonction $f(\varepsilon)$ peut s'écrire en première approximation

$$
f(\varepsilon)=V(\varepsilon) \cdot C(\varepsilon) \cdot H(\varepsilon)
$$

$V(\varepsilon)$ décrit le comportement viscoélastique sous la forme

$$
V(\varepsilon)=1-\exp \left(-w \cdot \varepsilon^{v}\right) .
$$

$C(\varepsilon)$ prend en compte un éventuel crochet de traction :

$$
C(\varepsilon)=1+a \cdot \exp (-b \cdot \varepsilon) .
$$

$H(\varepsilon)$ décrit le durcissement progressif aux grandes déformations :

$$
H(\varepsilon)=\exp \left(h \cdot \varepsilon^{n}\right)
$$

où l'exposant $n$ a une valeur voisine de 2 dans la plupart des cas.

La sensibilité à la vitesse, exprimée par la fonction $g(\dot{\varepsilon})$, peut se mettre sous la forme d'une loi puissance : $g(\dot{\varepsilon})=\dot{\varepsilon}^{m}$.

La dépendance de la contrainte aux variables $\varepsilon$ et $\dot{\varepsilon}$ est souvent exprimée sous forme des dérivées partielles du premier ordre [27]

$\gamma=(\partial \ln \sigma / \partial \varepsilon)_{\dot{\varepsilon}}$ coefficient de durcissement relatif $m=(\partial \ln \sigma / \partial \ln \dot{\varepsilon})_{\varepsilon}$ coefficient de sensibilité à la vitesse de déformation.
On peut constater que l'équation (4) sépare les variables $\varepsilon$ et $\dot{\varepsilon}$ sous forme multiplicative, de sorte que $\gamma$ ne dépendrait que de $\varepsilon$, et $m$ ne dépendrait que de $\dot{\varepsilon}$. Cette propriété est assez bien vérifiée dans la pratique en première approximation. Cependant, pour certaines applications, il est nécessaire de tenir compte des coefficients du second ordre : $A=(\partial \gamma / \partial \ln \dot{\varepsilon})_{\varepsilon}$ et $B=(\partial m / \partial \varepsilon)_{\dot{\varepsilon}}$. Ces influences croisées s'expriment essentiellement par de légères variations de $V(\varepsilon)$ et $C(\varepsilon)$ avec $\dot{\varepsilon}$ et de l'exposant $m$ avec $\varepsilon$.

A titre d'exemple, le Polyéthylène à haute densité possède une loi de comportement assez simple qui s'écrit :

$$
\sigma=K \cdot(1-\exp (-w \cdot \varepsilon)) \cdot \exp \left(h \cdot \varepsilon^{2}\right) \cdot \dot{\varepsilon}^{m}
$$

où les coefficients, à $22^{\circ} \mathrm{C}$, ont les valeurs suivantes : $K=46 \mathrm{MPa}, w=40, h=0,41, m \simeq 0.075$.

Il est intéressant de comparer ce comportement à celui d'un métal ductile (par exemple le cuivre). Dans ce cas, la loi de comportement est souvent écrite, [28], sous la forme :

$$
\sigma=K \cdot V(\varepsilon) \cdot\left(\varepsilon+\varepsilon_{0}\right)^{n} \cdot \dot{\varepsilon}^{m}
$$

où $V(\varepsilon)$ décrit le raccord élastique-plastique. Comme l'exposant $n$ est inférieur à $1(n \simeq 0,5$ pour le $\mathrm{Cu}$ à $22^{\circ} \mathrm{C}$ ), la pente de la courbe $\sigma(\varepsilon)$ dans le domaine plastique diminue progressivement alors qu'elle augmente dans le cas des polymères. Cette différence essentielle entre le comportement des deux types de matériaux est liée à la différence de leurs structures: mécanisme de restauration des dislocations dans les métaux, orientation progressive des chaînes dans les polymères.

\section{Manifestations de l'instabilité plastique dans les polymères en traction.}

La déformation plastique des polymères est un phénomène irréversible à court terme qui donne lieu à des instabilités à différentes échelles d'observation :

- à l'échelle microscopique (quelques nanomètres) : cascades de changements conformationnels des macromolécules

- à l'échelle mésoscopique (quelques micromètres): bandes de déformation et hétérogénéités intra-sphérolitiques

- à l'échelle macroscopique (quelques millimètres) : striction généralisée des éprouvettes.

Cette classification des phénomènes est nécessairement arbitraire car certaines instabilités se développent à plusieurs échelles simultanément. Elle permet toutefois d'analyser de manière plus rationnelle des faits expérimentaux complexes et variés. Nous en décrirons ci-dessous les principales manifestations. 
3.1 INSTABILITÉS MICROSCOPIQUES. - Dans les solides cristallins (métaux par exemple), la limite élastique s'accompagne souvent d'une avalanche de dislocations qui se multiplient à partir de sources localisées et se propagent dans un nombre limité de plans cristallographiques adjacents [29]. Ce mécanisme est très brutal si les obstacles au mouvement des dislocations (frottement de réseau, précipités, joints de grains...) sont peu efficaces. Il se forme ainsi des bandes de glissement qui constituent, à l'échelle microscopique, les premières manifestations de l'instabilité plastique.

Dans les polymères, des mécanismes du même type peuvent exister également, comme nous l'avons vu à la section 2.2. Des changements conformationnels coopératifs peuvent affecter toutes les molécules dans une zone plane soumise à une contrainte de cisaillement assez élevée. Si le polymère est cristallisé, le plan de cisaillement correspond nécessairement à un plan cristallographique contenant l'axe des chaînes. Si le polymère est amorphe, le plan de cisaillement n'est pas défini structuralement mais correspond simplement au plan où la contrainte de cisaillement (cission) est maximale. Il semble que, dans les polymères, le cisaillement plastique soit moins localisé que dans les autres matériaux pour deux raisons principales :

a) La nature macromoléculaire des chaînes polymères oppose une contrainte antagoniste au mouvement des dislocations. Dans les cristallites, cette contrainte est due au réajustement des repliements de chaînes à la surface ; dans les polymères vitreux, elle apparaît au niveau de chaque segment moléculaire subissant un changement de conformation et elle est due à l'effet entropique de l'orientation locale.

b) Le rapport $\tau_{y} / \mu$ de la cission critique de glissement plastique au module de cisaillement est beaucoup plus élevé dans les polymères $\left(\approx 10^{-1}\right)$ que dans les métaux $\left(\approx 10^{-5}\right)$. Il en résulte que la formation d'une zone lenticulaire cisaillée plastiquement au cœur d'un échantillon polymère soumis à une contrainte élastique est moins favorable que dans le métal car elle entraîne une relaxation d'énergie plus faible.

c) Des phénomènes de diffusion viscoélastique des chaînes peuvent relaxer, surtout au voisinage de $T_{\mathrm{g}}$, les gradients de déformation formés localement par la plasticité.

3.2 INSTABILITÉS MÉSOSCOPIQUES. - Le développement de gradients de déformation localisés dans des zones de quelques micromètres a fait l'objet de nombreuses publications.

Dans les polymères semi-cristallins, des hétérogénéités de déformation sont observées à l'intérieur de chaque sphérolite $[16,30]$. Dès que la limite élastique est amorcée, on assiste normalement à la déformation plastique par cisaillement des lamelles diagonales (Fig. 7b). Dans certains polymères [31], on assiste à une localisation catastrophique de la déformation avec allongement extrême du sphérolite, fragmentation des lamelles et réorientation des chaînes dans le sens de l'étirage (Fig. 7c). Dans ce mécanisme, la phase amorphe caoutchoutique agit comme un liant souple et accommode les incompatibilités de déformation entre les sphérolites. Il est donc assez rare de voir apparaître des bandes de déformation se propageant au travers de plusieurs sphérolites.

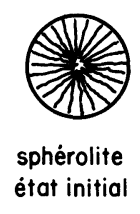

a

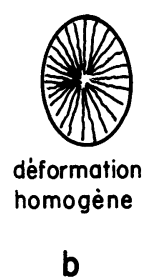

b

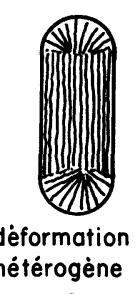

C
Fig. 7. - Instabilité plastique intra-sphérolitique dans un polymère semi-cristallin.

[Intra-spherulitic plastic instability in a semi-crystalline polymer.]

Le comportement des polymères vitreux est très différent. La littérature rapporte de nombreuses observations de bandes de déformation localisées. Bien que la plupart de ces travaux concernent des expériences de compression (par exemple Réf. [23]), les bandes de déformation se forment également en traction [32-37], à condition qu'un phénomène de rupture prématurée ne se produise pas avant l'écoulement plastique (comme dans PS et PMMA à l'ambiante). Pour tous les polymères vitreux, le mécanisme de formation des bandes est similaire. Pour une déformation très faible (quelques pourcent), des zones de déformation localisées se forment dans les parties les plus sollicitées de l'éprouvette : entailles, zones à concentrations de contrainte, inclusions... Leur épaisseur et leur vitesse de propagation sont très variables selon le matériau, la température et la géométrie des éprouvettes. A la température ambiante, des bandes de déformation de quelques dizaines de micromètres d'épaisseur ont été rapportées dans le PET, le PVC et le PC. Dans les premiers stades d'évolution du phénomène, la déformation associée à ces bandes est un cisaillement simple presque parfait. La direction du plan de cisaillement est sensiblement parallèle au plan de cission maximum. Des écarts à cette direction sont observés dans les polymères dont la cission critique de glissement dépend de la contrainte normale au plan de cisaillement (critère de Mohr-Coulomb, Réf. [38-39]). Pour des étirages assez lents (Fig. 8) la 

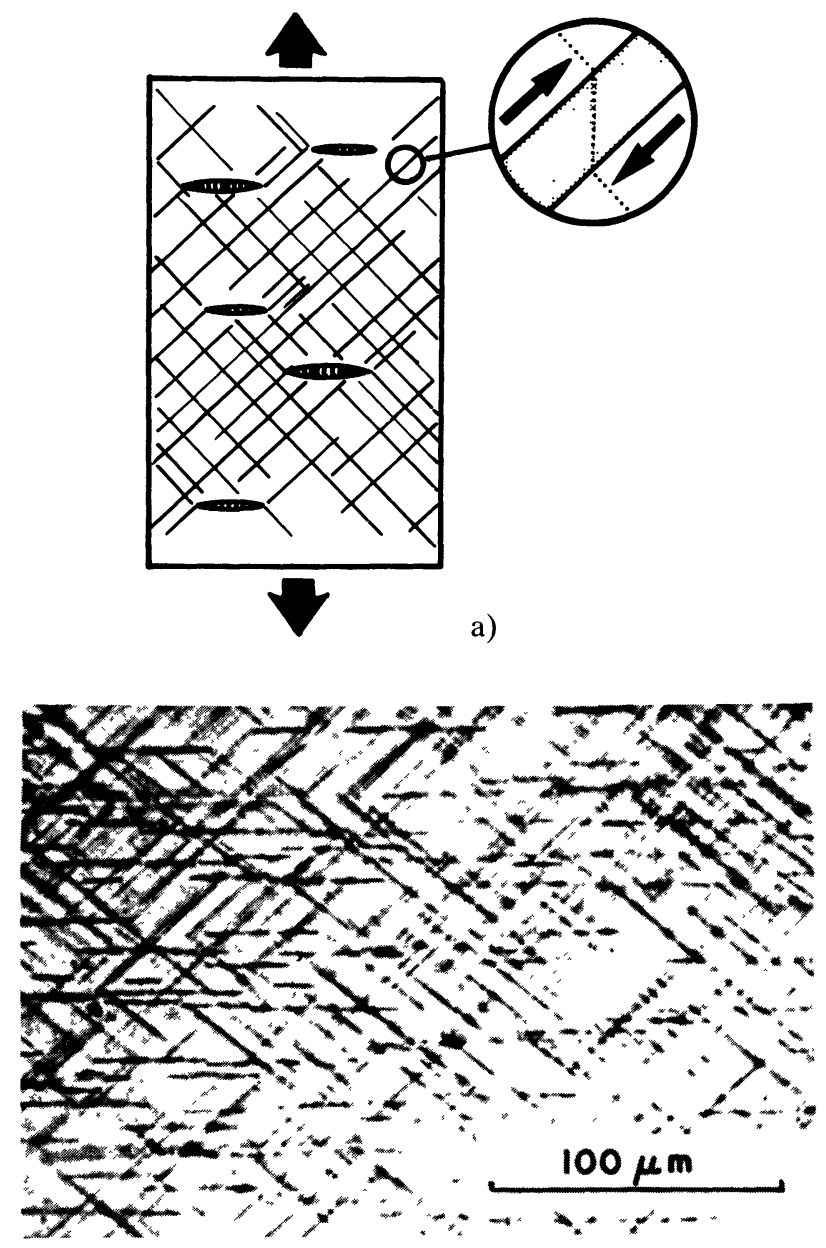

b)

Fig. 8. - Formation de bandes de cisaillement à la pointe des microcraquelures dans un polymère vitreux, a) schéma de principe ; b) observation dans un film de PET.

[Formation of shear bands at the tip of crazes in a polymer, a) schematic diagram; b) observation in a PET film.]

plasticité par bandes de cisaillement mésoscopiques peut accompagner la formation de microcraquelures comme dans le PET amorphe [5] ; dans ce cas, les bandes sont formées aux extrémités des craquelures. La valeur du cisaillement à l'intérieur d'une bande dépend du matériau et des conditions expérimentales : il est généralement compris entre $\gamma_{B}=0,5$ et 1,0 dans les polymères vitreux ductiles à l'ambiante, mais cette valeur décroît au voisinage de $T_{\mathrm{g}}$. L'évolution des bandes de cisaillement au cours d'un étirage est complexe. Les bandes se multiplient et se propagent en longueur comme en épaisseur afin d'accommoder l'allongement imposé à l'éprouvette. Il faut bien considérer que l'essai de traction (ou de compression) n'est pas bien adapté à une étude fine de la cinétique de croissance des bandes de cisaillement car les deux modes ne sont compatibles qu'aux faibles déformations. Pour analyser cette cinétique avec précision, des essais de cisaillement simple sont beaucoup mieux adaptés et permettent des observations directes des bandes de cisaillement en cours d'évolution [40-41]. Dans un essai de traction avec une éprouvette géométriquement homogène et bien guidée axialement (par exemple une éprouvette ronde bien polie) le développement des bandes se produit de manière très diffuse (Fig. $9 \mathrm{a})$. Au contraire, si l'éprouvette présente des imperfections géométriques ou structurales asymétriques et si ses têtes ont une certaine liberté de mouvement latéral, le glissement se concentre autour d'un plan oblique particulier et on voit se former une sorte de bande de cisaillement très épaisse qui traverse l'échantillon d'un côté à l'autre (Fig. 9b). A partir de ce moment, la bande devient le siège de déformations triaxiales généralisées avec réduction d'épaisseur et allongement perpendiculairement au plan de la bande. Sa direction tend alors à s'éloigner de l'axe de traction, comme dans le cas des bandes de Lüders. Cette analyse a fait l'objet d'études mécaniques détaillées [42-44].

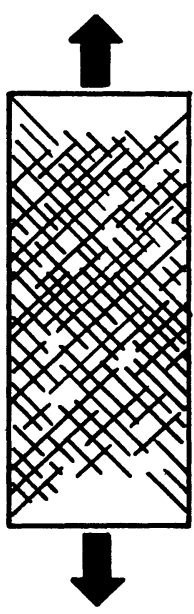

a)

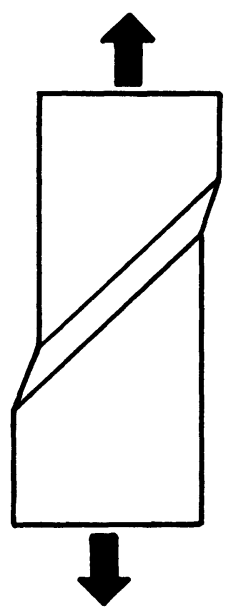

b)
Fig. 9. - Bandes de cisaillement diffuses ou localisées au cours de la traction d'un polymère vitreux.

[Diffuse or localized shear bands during the tensile testing of a glassy polymer.]

Dans un cas comme dans l'autre, le développement des instabilités plastiques mésoscopiques conduit à des instabilités macroscopiques aux grands allongements. La prolifération régulière des bandes de cisaillement dans une zone légèrement plus faible de l'éprouvette aboutit à la formation d'une striction diffuse ; au contraire, l'épaississement exagéré d'une bande de cisaillement amène la formation d'une striction oblique localisée avec un éventuel pliage si les têtes de l'éprouvette peuvent tourner.

3.3 InSTABILITÉS MACROSCOPIQUes. - Nous nous intéresserons dans cette partie au cas de la striction 
diffuse, ou striction généralisée. Ce mode d'instabilité plastique se distingue des bandes de cisaillement et des strictions obliques localisées (Fig. 9) dans la mesure où il respecte localement le champ de déformation général de l'éprouvette. C'est ainsi qu'un quadrillage orthogonal tracé sur la surface d'une éprouvette à l'état d'origine conserve sensiblement son orthogonalité après striction diffuse (pour autant que le gradient de déformation soit assez faible) alors que le même quadrillage présenterait un cisaillement important et éventuellement hétérogène si l'éprouvette subissait une instabilité oblique.

Deux cas limites sont particulièrement intéressants à examiner : celui de l'étirage d'un barreau cylindrique (traction uniaxiale) et celui de l'étirage d'un film à largeur fixe (traction en déformation plane).

Le premier cas est illustré à la figure 10 par l'étirage d'un barreau cylindrique de Polyéthylène à haute densité (HDPE) de diamètre $8 \mathrm{~mm}$ contenant initialement en son centre un défaut de section de l'ordre de $4 \%$. La striction se forme très tôt $\left(\Delta L / L_{0} \simeq 5\right.$ à $\left.10 \%\right)$ dès le début de la plasticité. Elle s'aggrave rapidement, tandis que la force de traction décroît, jusqu'à ce que la déformation vraie

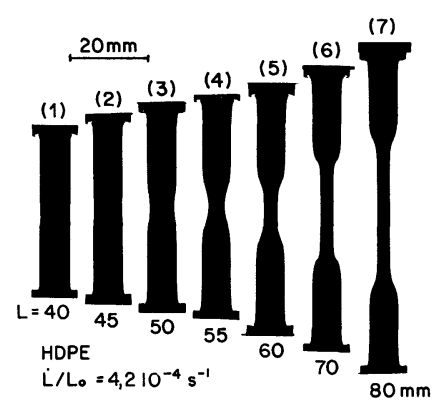

a)

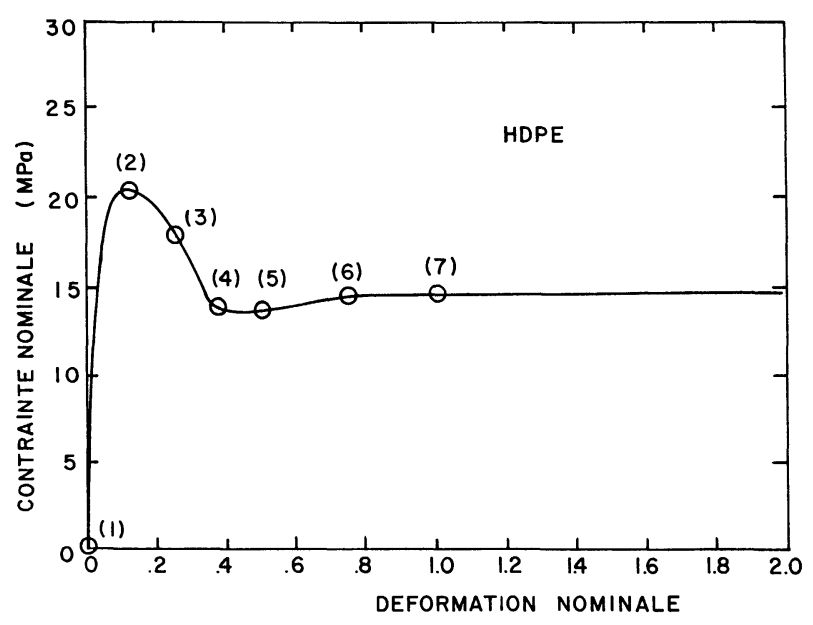

b)

Fig. 10. - Formation et propagation d'une striction dans un barreau cylindrique de Polyéthylène à Haute Densité.

[Initiation and propagation of a neck in a cylindrical bar of High Density Polyethylene.] au centre du rétrécissement soit voisine de 1,2. Ensuite, contrairement au comportement des métaux, la striction se stabilise et se propage tout au long de l'éprouvette. Pendant toute cette phase, la force d'étirage est sensiblement constante, de même que la forme des épaules de la striction, et le gradient de déformation maximum est assez modéré. Le taux d'allongement dans la partie étirée (appelée taux d'étirage naturel) reste pratiquement constant. On assiste donc à un effet mécanique remarquable : la propagation d'un régime de déformation à 2 états (état non déformé, état déformé) avec une zone de transition bien localisée qui se translate à vitesse constante. On a pu comparer ce phénomène à celui de l'écoulement continu de la matière dans un convergent sans parois [45]. Il est mis à profit dans certains procédés d'étirage industriels où l'on désire auto-renforcer un polymère par orientation des chaînes. Il est clair en effet que c'est cette orientation (et l'augmentation de la contrainte d'écoulement qui lui est associée) qui provoque la stabilisation de la déformation au centre du défaut. Il faut prendre garde cependant aux effets anisothermes: si la vitesse d'étirage est élevée, la chaleur dégagée pour la déformation plastique dans la striction n'a plus le temps de diffuser et le matériau peut subir une rupture thermique [46] par ramollissement local du polymère.

Le second cas examiné ici (Fig. 11) est celui de l'étirage d'un film de Polytérephtalate d'éthylène glycol (PET) d'épaisseur $150 \mu \mathrm{m}$ soumis à un étirage à largeur fixe (imposée par le très grand rapport largeur/longueur $=80 \mathrm{~mm} / 10 \mathrm{~mm}$ de l'éprouvette). Dans ce cas, on a un état de déformation plane et on montre aisément [42] qu'une instabilité plastique macroscopique ne peut se former que le long d'une direction perpendiculaire à l'axe d'étirage. Afin d'amener cette striction à apparaître au centre de l'éprouvette (et non pas le long des pinces de serrage) l'éprouvette de film a été munie initialement d'un léger défaut mécanique central (produit par pliage). On observe bien la formation d'une striction dans cette zone. Comme dans le Polyéthylène, la striction du PET se stabilise après une certaine déformation et se propage à force constante. Une coupe réalisée perpendiculairement au film (Fig. 11c) montre bien que le profil d'une épaule de la striction a une forme analogue à celle observée à une échelle 40 fois plus grande dans le barreau de Polyéthylène. Le fait que le $\mathrm{PET}$ soit un polymère amorphe vitreux n'apparaît pas de manière évidente à l'échelle macroscopique. En revanche, l'observation fine de la striction au microscope électronique à balayage (échelle mésoscopique) révèle la présence de nombreuses bandes de cisaillement qui émergent à la surface dans cette zone (Fig. 12). Cette observation montre bien la continuité entre les notions d'instabilités mésoscopique et macroscopique. 


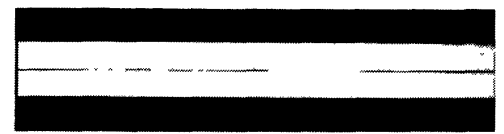

(1)
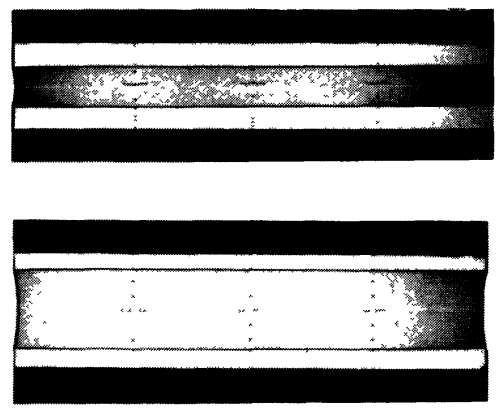

(3)

$20 \mathrm{~mm}$

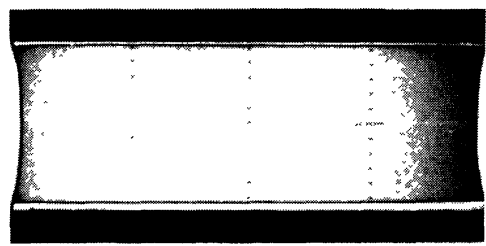

(4)

a)

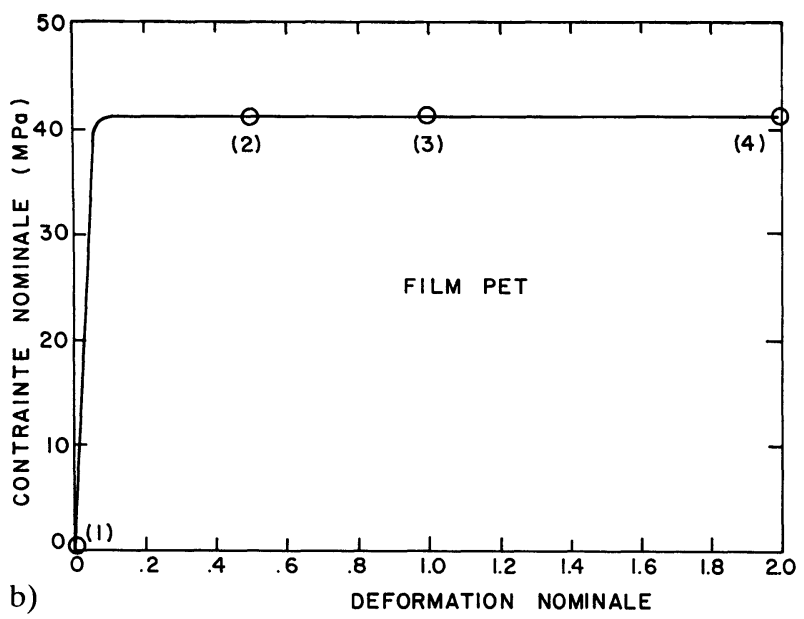

c)

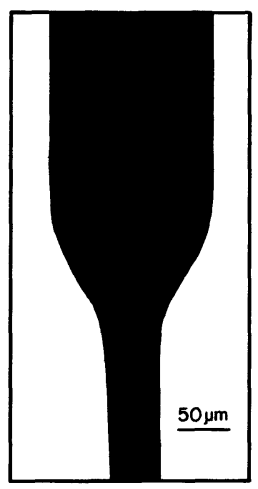

Fig. 11. - Formation et propagation d'une striction dans un film mince de Polyéthylène Terephtalate (PET).

[Initiation and propagation of a neck in a thin film of Polyethylene Terephtalate (PET), a) front view of the sample during the tensile test; b) nominal stress $v s$. nominal strain curve; c) side view of the film profile at the upper limit of the neck.]
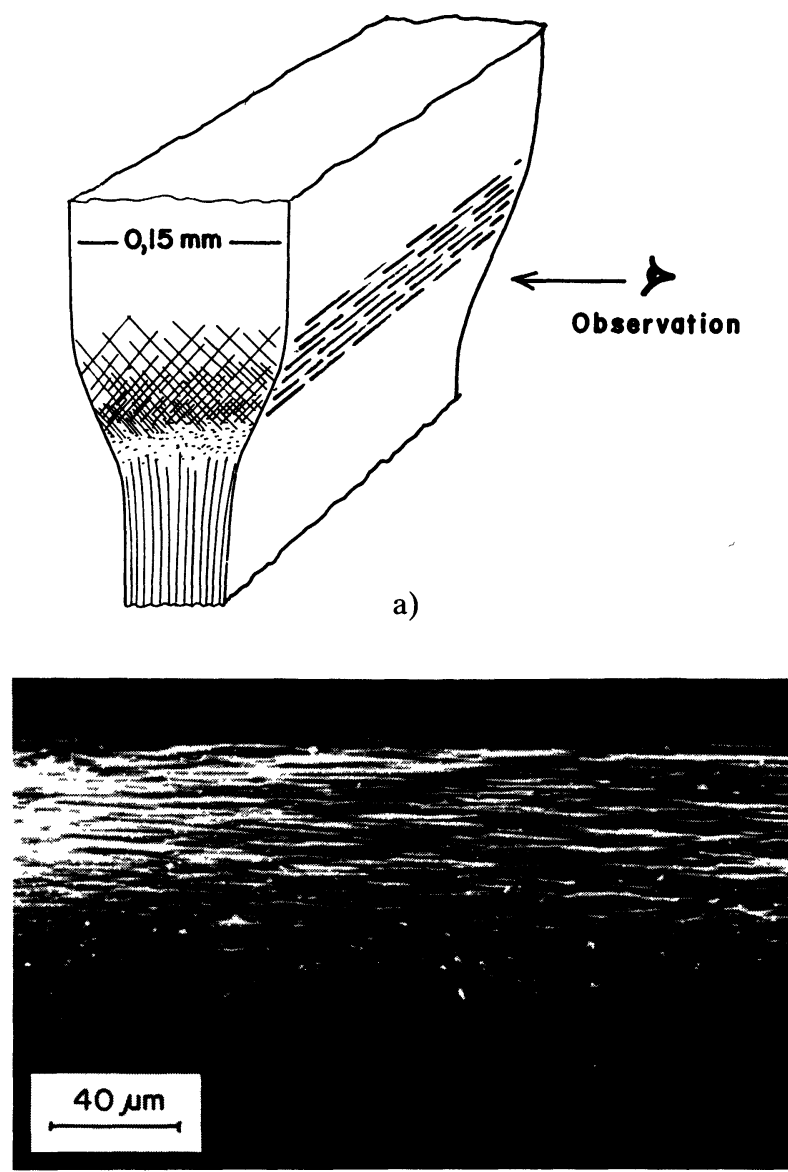

b)

Fig. 12. - Emergences de bandes de cisaillement mésoscopiques à la limite de la striction dans un film mince de Polytérephtalate d'Ethylène Glycol (PET), a) conditions d'observation ; b) micrographie électronique à balayage.

[Emergence of microscopic shear bands at the limit of the neck in a thin films of PET, a) observation conditions ; b) Scanning Electron Microscopy.]

\section{Analyse théorique de l'instabilité plastique en traction.}

De nombreux travaux fondamentaux ont été consacrés au cours de la dernière décennie à l'interprétation et à la modélisation des phénomènes d'instabilité plastique dans les matériaux soumis à un étirage (pour une revue de ces travaux, voir [2]). Un intérêt particulier a été porté : i) aux conditions d'apparition des bandes de cisaillement mésoscopiques et, ii) au développement de la striction diffuse dans un barreau axisymétrique ou dans une plaque fine. La plupart de ces travaux ont porté sur les métaux et leurs conclusions sont adaptées à leur loi de comportement. Dans le cas des polymères, l'étude des instabilités d'étirage est plus récente et doit encore progresser. Comme nous allons le voir ci-dessous, les méthodes d'analyse mises au point pour les métaux sont généralement applicables mais leurs 
conclusions diffèrent lorsque l'on prend en compte les lois de comportement propres aux polymères.

\subsection{FORMATION D'UNE BANDE DE CISAILLEMENT.} A l'échelle mésoscopique, une bande de cisaillement est caractérisée par la présence d'un gradient de déformation en cisaillement par rapport au reste de l'échantillon. La modélisation du développement de telles bandes dans des échantillons en traction est un problème assez difficile: plusieurs systèmes de bandes apparaissent sur des familles de plans différentes, se croisent, se dévient mutuellement, etc... De plus, si l'échantillon est assez mince, le champ de déformation au sein d'une bande donnée échappe rapidement aux conditions idéales du cisaillement simple.

Plusieurs auteurs [47-49] ont analysé les critères d'apparition des bandes de cisaillement dans un métal ou un polymère en simplifiant le problème géométrique : ils ont considéré le cas d'un élément de matière parallélèpipédique soumis à un cisaillement simple macroscopique avec une vitesse de cisaillement appliquée constante $\dot{\varepsilon}_{12} \quad$ (Fig. 13).

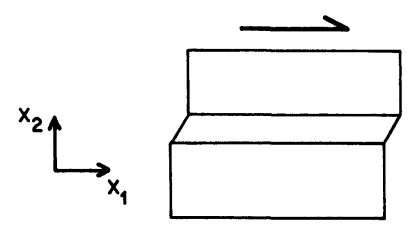

Fig. 13. - Cas simplifié de la formation d'une bande de cisaillement dans un échantillon soumis à un cisaillement simple macroscopique.

[Simplified case of shear banding in a sample subjected to macroscopic simple shear.]

L'échantillon contient localement une imperfection de section ou de structure qui favorise la localisation du cisaillement dans un plan donné. Ces travaux ont montré qu'une bande de cisaillement ne peut se former que si l'une des deux conditions suivantes est vérifiée :

a) la loi de comportement intrinsèque du matériau en cisaillement simple présente une phase d'adoucissement plastique (pente négative de la courbe $\left.\sigma_{12}\left(\varepsilon_{12}\right)\right)$. Cela est vérifié dans le cas des polymères vitreux au moment de la limite élastique du fait que le déclenchement de la plasticité est plus difficile que sa continuation.

b) La déformation plastique s'accompagne d'un adoucissement thermique à cause de l'auto-échauffement du matériau. Cela est particulièrement sensible dans le cas des déformations rapides quand la chaleur dégagée par les processus moléculaires de déformation ne diffuse pas assez rapidement dans la matière environnante. Le cas des polymères est critique (surtout les amorphes) car leur condition thermique est particulièrement faible par rapport aux métaux.

Une fois formées, les bandes de cisaillement mésoscopiques se développent plus ou moins selon les paramètres de la loi de comportement plastique du matériau en cisaillement simple. Si le taux de durcissement plastique est élevé, le cisaillement dans la bande se stabilise rapidement et la déformation de l'échantillon se poursuit par épaississement de la bande initiale ou par formation de bandes secondaires. Si la sensibilité du matériau à la vitesse de déformation est grande, la formation de la bande est très ralentie, à tel point que dans certains polymères (au voisinage de $T_{\mathrm{g}}$ par exemple), les bandes de cisaillement ne sont même plus observables.

4.2 FORMATION D'UNE STRICTION DIFFUSE : ANALYSE UNIAXIALE. - Les conditions dans lesquelles une striction se développe dans un échantillon polymère en traction ont fait l'objet de plusieurs études approfondies [5, 12, 45, 50-54]. Un cas typique est celui d'un barreau cylindrique de Polyéthylène contenant un défaut géométrique initial (illustré à la Fig. 10). L'analyse la plus simple consiste à considérer que le champ de contraintes est purement uniaxial en tout point de l'éprouvette, repéré par sa coordonnée $X$ le long de l'axe. En négligeant les effets d'inertie (notables seulement aux très fortes vitesses), on a donc

$$
F=\sigma(X) \cdot A(X)=\text { Const. }
$$

où $F$ est la force de traction, $A$ la section courante de l'éprouvette et $\sigma$ mesure à la fois la contrainte axiale $\sigma_{X X}$ et la contrainte effective $\sigma_{\text {eff }}$ car les composantes radiale $\left(\sigma_{\mathrm{rr}}\right)$ et circonférentielle $\left(\sigma_{\theta \theta}\right)$ sont supposées nulles en première approximation. Dans cette analyse, la coordonnée $X$ est considérée comme la cote Lagrangienne du point courant, c'est-à-dire qu'elle est liée à un élément de matière. La distance Lagrangienne $\delta X$ entre deux éléments voisins ne varie donc pas au cours de la déformation, au contraire de la distance Eulérienne $\delta x$ (coordonnée normale du laboratoire) qui augmente avec la déformation vraie locale $\varepsilon$ selon l'expression $\delta x=$ $\delta X \cdot \exp (\varepsilon)$. L'analyse en coordonnées Eulériennes est également possible [52] mais introduit des termes convectifs supplémentaires assez lourds. Sous forme différentielle, l'équation (8) s'écrit aussi, à chaque instant $t$

$$
\left[\frac{\delta \ln \sigma(X)}{\delta X}\right]_{t}+\left[\frac{\delta \ln A(X)}{\delta X}\right]_{t}=0 .
$$

La variation de la contrainte vraie $\sigma$ le long de l'éprouvette est liée aux variations de la déformation $\varepsilon$, de la vitesse de déformation $\dot{\varepsilon}$ et de la température 
$T$. On peut écrire, en supposant l'existence d'une équation d'état $\sigma(\varepsilon, \dot{\varepsilon}, T)$

$$
\begin{aligned}
{\left[\frac{\delta \ln \sigma}{\delta X}\right]_{t}=\left[\frac{\partial \ln \sigma}{\partial \varepsilon}\right]_{\dot{\varepsilon}, T} \cdot\left[\frac{\delta \varepsilon}{\delta X}\right]_{t} } \\
+\left[\frac{\partial \ln \sigma}{\partial \ln \dot{\varepsilon}}\right]_{\varepsilon, T} \cdot\left[\frac{\delta \ln \dot{\varepsilon}}{\delta X}\right]_{t} \\
+\left[\frac{\partial \ln \sigma}{\partial T}\right]_{\varepsilon, \dot{\varepsilon}} \cdot\left[\frac{\delta T}{\delta X}\right]_{t}
\end{aligned}
$$

que l'on peut écrire aussi

$$
\begin{aligned}
{\left[\frac{\delta \ln \sigma}{\delta X}\right]_{t}=\gamma \cdot\left[\frac{\delta \varepsilon}{\delta X}\right]_{t}+m } & \cdot\left[\frac{\delta \ln \dot{\varepsilon}}{\delta X}\right]_{t}+ \\
& +\Phi \cdot\left[\frac{\delta T}{\delta X}\right]_{t}
\end{aligned}
$$

où $\gamma$ et $m$ sont les coefficients de durcissement et de sensibilité à la vitesse (déjà définis en 2.3.3) et $\Phi=(\partial \ln \sigma / \partial T)_{\varepsilon, \dot{\varepsilon}}$ mesure la sensibilité de la contrainte d'écoulement plastique à la température (avec $\Phi<0$ ). Le deuxième terme de l'équation (9) est lié d'une part aux variations de la section initiale de l'éprouvette $A_{0}(X)$, et d'autre part aux variations de la déformation courante

$$
\left[\frac{\delta \ln A}{\delta X}\right]_{t}=\frac{\mathrm{d} \ln A_{0}}{\mathrm{~d} X}-\left[\frac{\delta \varepsilon}{\delta X}\right]_{t} .
$$

On définit le Gradient de Déformation $\lambda$ comme la dérivée locale de la déformation par rapport à la coordonnée Lagrangienne $X$ à un instant donné $t$ selon l'équation

$$
\lambda=\left[\frac{\delta \varepsilon}{\delta X}\right]_{t} .
$$

Il est intéressant de remarquer que dans l'équation (11), le second terme du deuxième membre (qui exprime le gradient de vitesse) peut s'écrire également

$$
\left[\frac{\delta \ln \dot{\varepsilon}}{\delta X}\right]_{t}=\frac{1}{\dot{\varepsilon}}\left[\frac{\delta \dot{\varepsilon}}{\delta X}\right]_{t}=\frac{\mathrm{d}}{\mathrm{d} \varepsilon}\left[\frac{\delta \varepsilon}{\delta X}\right]_{t}=\left[\frac{\delta \lambda}{\delta \varepsilon}\right]_{t} .
$$

(Note : en coordonnées Eulériennes, la manipulation ci-dessus n'est pas correcte et il est nécessaire d'introduire des termes supplémentaires.)

En réunissant les équations (10) à (14), on arrive finalement à l'équation suivante

$m \cdot\left[\frac{\delta \lambda}{\delta \varepsilon}\right]_{t}=(1-\gamma) \cdot \lambda-\Phi \cdot\left[\frac{\delta T}{\delta X}\right]_{t}-\frac{\mathrm{d} \ln A_{0}}{\mathrm{~d} X}$.

Considérons tout d'abord le cas d'un essai de traction très lent, réalisé dans des conditions isothermes idéales, avec $(\delta T / \delta X)_{t}=0$. On constate alors que (15) constitue une équation différentielle simple qui régit les variations du gradient de déformation $\lambda$ en fonction de la déformation courante $\varepsilon$ pour une valeur du gradient de section initiale $\left(\mathrm{d} \ln A_{0} / \mathrm{d} X\right)$. On a vu à la section 2.3.3 que le coefficient $m$ pouvait être considéré constant en première approximation ( $m \simeq 0,075$ pour HDPE). Le coefficient de durcissement relatif $\gamma$ est fonction de $\varepsilon$ puisque, d'après (6) on a

$$
\gamma(\varepsilon)=\frac{w}{\exp (w \varepsilon)-1}+2 h \cdot \varepsilon .
$$

Les variations de $\gamma$ avec $\varepsilon$ sont illustrées à la figure 14. A partir de ces équations de base, considérons le cas d'un élément de matière situé dans la zone du défaut géométrique. En choisissant la direction de l'axe des coordonnées $X$ vers le centre du défaut, on a alors $-\left(\mathrm{d} \ln A_{0} / \mathrm{d} X\right)>0$. Au début de l'étirage, la déformation est nulle partout, d'où $\lambda \equiv 0$. A $t=0$, on a donc

$$
(\delta \lambda / \delta \varepsilon)_{0}=-(1 / m) \cdot\left(\mathrm{d} \ln A_{0} / \mathrm{d} X\right)>0 .
$$

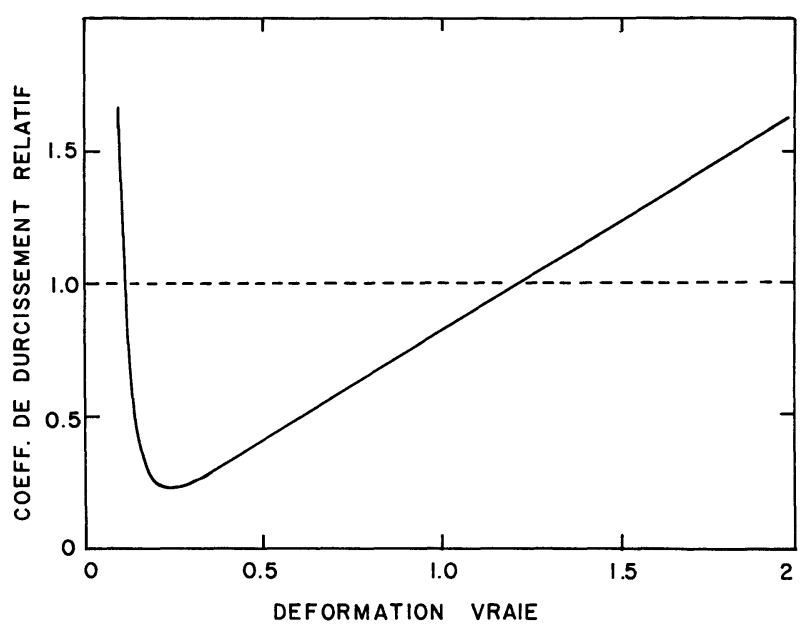

Fig. 14. - Variation du coefficient de durcissement relatif $\gamma=(\partial \ln \sigma / \partial \varepsilon)_{\dot{\varepsilon}, \mathrm{T}}$ du PEHD avec la déformation.

[Evolution with strain of the relative strain-hardening coefficient $\gamma=(\partial \ln \sigma / \partial \varepsilon)_{\dot{\varepsilon}, \mathrm{T}}$ of High Density Polyethylene.]

Dès le début de l'essai, la fluctuation initiale de section tend à s'amplifier : la zone à section réduite se creuse un peu plus. Toutefois, cette évolution initiale tend à se stabiliser tant que $\gamma>1$ car, dans l'équation différentielle (15), le coefficient de $\lambda$ devient alors négatif. Cette évolution stable du défaut géométrique dure jusqu'à ce que $\varepsilon$ atteigne une valeur de quelques pour cent, quant $\gamma$ passe en dessous de la valeur critique $\gamma=1$ (Fig. 14). La tendance change alors en une localisation plus intense du défaut et $\lambda$ subit une croissance de plus en plus rapide : c'est le stade d'instabilité auto-accélérée qui correspond au développement visible de la 
striction, tel qu'on l'observe expérimentalement (Fig. 10). On voit bien d'après (15) que la croissance du gradient de déformation est d'autant plus rapide que le coefficient $m$ est plus petit. Ainsi dans le PET $(m \simeq 0,03)$, la striction est plus rapide que dans le Polyéthylène $(m \approx 0,075)$. L'intégration numérique de l'équation (15) à température constante et pour une valeur donnée de $\mathrm{d} \ln A_{0} / \mathrm{d} X$ conduit à une courbe $\lambda(\varepsilon)$ en forme de cloche (Fig. 15). On constate que le stade de croissance instable de $\lambda$ ne se prolonge pas jusqu'à rupture de l'éprouvette (comme dans les métaux ductiles) mais s'arrête à une déformation voisine de $\varepsilon \simeq 1,2$, après quoi $\lambda$ diminue rapidement. Cette propriété particulière des polymères est liée à l'augmentation progressive du coefficient de durcissement $\gamma$ aux grandes déformations, associée à la rotation des chaînes macromoléculaires vers la direction d'étirage. On assiste alors à une stabilisation de la striction : la déformation n'y augmente pratiquement plus et c'est le tour des zones extérieures au défaut géométrique de se déformer à leur tour.

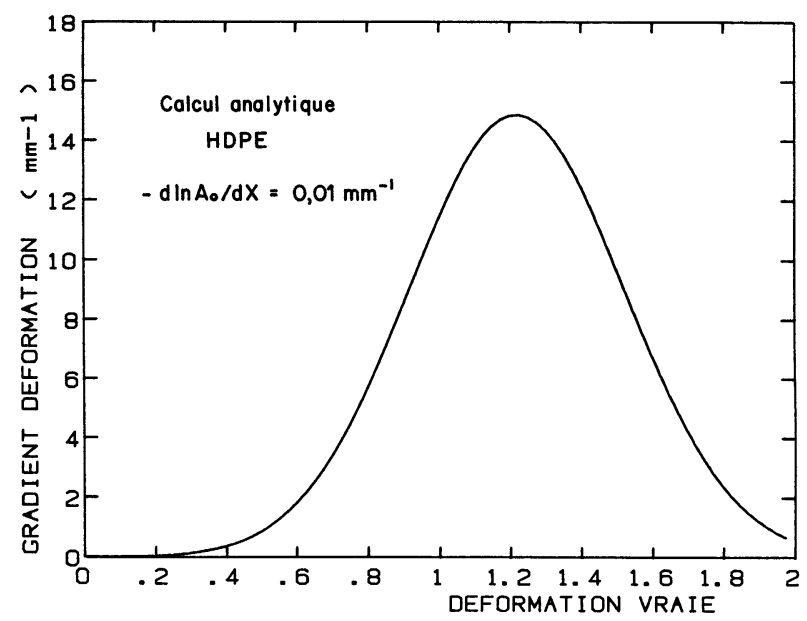

Fig. 15. - Evolution du gradient de déformation au cours de l'étirage à partir d'un défaut géométrique (courbe obtenue par intégration numérique de l'équation (15)).

[Evolution of strain gradient from a geometric defect during stretching (deduced by numerical integration of equation (15)).]

On peut donc constater que la simple exploitation de la loi de comportement du matériau (Eq. (6)) et de la condition d'équilibre mécanique de l'éprouvette (Eq. (8)) permettent de rendre compte qualitativement des principaux traits du phénomène de striction dans le Polyéthylène. Si l'on tient compte en plus de l'adoucissement thermique qui apparaît aux grandes vitesses à cause de l'auto-échauffement de l'éprouvette (second terme du deuxième membre de l'équation (15)) on interprète facilement l'accélération de la striction qui est associée à cet effet.
Toutefois, l'analyse uniaxiale que nous avons présentée ci-dessus n'est pas satisfaisante à deux points de vue :

- l'intégration de l'équation (15) pour des valeurs usuelles de $\left(\mathrm{d} \ln A_{0} / \mathrm{d} X\right)$ conduit à des valeurs beaucoup trop fortes du gradient de déformation. Par exemple, les valeurs atteintes par $\lambda$ dans l'exemple de la figure 15 sont hors de proportion avec les valeurs expérimentales de $\lambda$ qui sont toujours inférieures à $1 \mathrm{~mm}^{-1}$. Il semble donc que l'équation (15) omette un paramètre qui aurait pour effet d'atténuer systématiquement l'instabilité plastique ;

- l'analyse uniaxiale rend bien compte de la stabilisation de la striction mais pas du phénomène de propagation régulière. Les observations expérimentales (Fig. 10) montrent que les zones en dehors du défaut géométrique ne se déforment pas en bloc après la stabilisation de la striction mais qu'une onde de déformation plastique se propage vers les extrémités de l'éprouvette d'une manière très progressive. En aucune façon, l'analyse uniaxiale présentée plus haut ne permet de rendre compte de ce processus. Eventuellement, le phénomène d'auto-échauffement pourrait entraîner une onde de déformation en même temps qu'il engendre une onde de chaleur ; toutefois, la littérature rapporte que la propagation de la striction est encore visible à des vitesses infinitésimales où l'échauffement adiabatique est négligeable [55]. Comme nous allons le voir cidessous, il est donc nécessaire d'introduire un nouvel ingrédient dans la théorie.

\subsection{EFFET DE LA TRIAXIALITÉ DES CONTRAINTES} SUR LE DÉVELOPPEMENT DE LA STRICTION. - Une analyse plus approfondie du phénomène de striction [51-53] montre que les composantes non axiales du tenseur des contraintes cessent d'être négligeables dès que la courbe relative $R / R_{\mathrm{c}}$ du profil de l'éprouvette devient notable au niveau de la striction ( $R$ est le rayon de l'éprouvette et $R_{\mathrm{c}}$ le rayon de courbure local). Qualitativement, il apparaît qu'une courbure concave (par exemple au centre de la striction) provoque le développement de termes $\sigma_{\mathrm{rr}}$ et $\sigma_{\theta \theta}$ positifs alors qu'une courbure convexe (aux épaules de la striction) les rend négatifs. Si l'on suppose que les polymères obéissent aux équations de plasticité de Von Mises [56], l'effet des contraintes non axiales est de modifier la contrainte effective $\sigma_{\text {eff }}$ (second invariant du tenseur des contraintes) qui contrôle la déformation généralisée du matériau (Fig. 16). Si $\sigma_{\mathrm{rr}}$ et $\sigma_{\theta \theta}$ sont positifs (courbure concave), la contrainte $\sigma_{\text {eff }}$ est inférieure à $\sigma=\sigma_{X X}$, ce qui entraîne une certaine stabilisation de l'instabilité plastique. A la limite du défaut, au contraire, la courbure est convexe et $\sigma_{\text {eff }}$ est supérieure à $\sigma_{X X}$ : on a alors accélération locale de la déformation, ce qui entraîne la propagation de la 


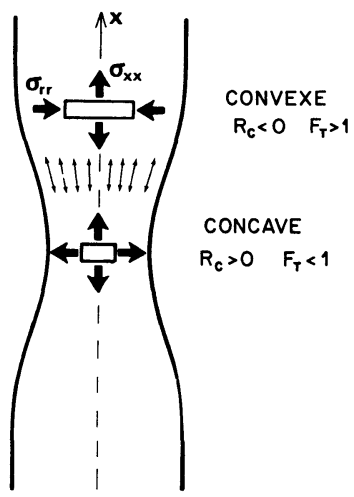

Fig. 16. - Représentation schématique des contraintes transversales engendrées par la courbure locale du profil d'une éprouvette de traction.

[Schematic diagram of transversal stresses induced by the local curvature of the sample profile.]

strịction vers les extrémités de l'éprouvette. Il semble donc bien que l'effet de triaxialité soit en mesure de rendre compte des insuffisances de l'analyse uniaxiale.

D'un point de vue quantitatif, le problème est plus complexe. La littérature offre plusieurs descriptions des contraintes transversales dans une striction axisymétrique, mais toutes reposent sur des hypothèses simplificatrices. L'analyse la plus couramment utilisée est celle de Bridgman [24] qui exprime l'effet des contraintes transversales par un paramètre scalaire unique $F_{\mathrm{T}}=\sigma_{\text {eff }} / \sigma_{X X}$, appelé Facteur de Triaxialité, qui ne dépend que du paramètre géométrique $R / R_{\mathrm{c}}$. Nous avons déjà utilisé la formule de Bridgman dans l'équation (3) pour la correction de contrainte vraie au centre de la striction, mais maintenant, nous l'appliquons tout le long de l'éprouvette. Les variations de $F_{\mathrm{T}}\left(R / R_{\mathrm{c}}\right)$ sont illustrées à la figure 17. Malgré les hypothèses très simples qui sous-tendent le calcul du facteur $F_{\mathrm{T}}$, sa validité opérationnelle a été confirmée par une analyse détaillée du tenseur des contraintes dans le

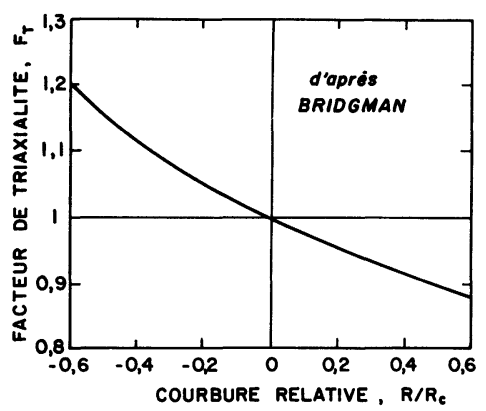

Fig. 17. - Variation du Facteur de Triaxialité de Bridgman $F_{\mathrm{T}}$ avec la courbure relative du profil $R / R_{\mathrm{c}}$.

[Evolution with the relative curvature $R / R_{\mathrm{c}}$, of the Bridgman triaxiality Factor $F_{\mathrm{T}}$.]
Polyéthylène à l'aide de la méthode des éléments finis [53]: la formule de Bridgman semble sousévaluer un peu l'effet de triaxialité pour les valeurs négatives de $R / R_{\mathrm{c}}$ mais les variations de la courbe de la figure 17 sont bien respectées en première approximation.

Dans l'approche analytique de la striction, l'introduction du facteur de triaxialité $F_{\mathrm{T}}$ revient à remplacer l'équation (8) par la nouvelle formulation

$$
F=\frac{\sigma_{\text {eff }}(X)}{F_{\mathrm{T}}(X)} \cdot A(X)=\text { Const. }
$$

L'équation d'état du matériau s'écrit maintenant $\sigma_{\text {eff }}\left(\varepsilon_{\text {eff }}, \dot{\varepsilon}_{\text {eff }}\right)$ où l'on suppose que $\varepsilon_{\text {eff }} \simeq \varepsilon$. On obtient ainsi l'équation différentielle régissant l'évolution de $\lambda(\varepsilon)$

$$
\begin{array}{r}
m \cdot\left[\frac{\delta \lambda}{\delta \varepsilon}\right]_{t}=(1-\gamma) \cdot \lambda-\Phi \cdot\left[\frac{\delta T}{\delta X}\right]_{t} \\
-\frac{\mathrm{d} \ln A_{0}}{\mathrm{~d} X}+\left[\frac{\delta \ln F_{\mathrm{T}}}{\delta X}\right]_{t}
\end{array}
$$

où le gradient de triaxialité $\left(\delta \ln F_{\mathrm{T}} / \delta X\right)$ joue le rôle d'amortisseur et de propagateur de la striction. Malheureusement, cette équation ne peut plus être intégrée simplement comme l'équation (15) car $F_{\mathrm{T}}$ dépend de la dérivée seconde du profil de l'éprouvette, et non plus seulement du gradient de déformation local $\lambda$.

Un moyen efficace de modélisation de la striction utilisant la notion de Facteur de Triaxialité est celle de la Méthode des Différences Finies [50,57]. Dans cette approche, l'échantillon est discrétisé géométriquement sous forme d'éléments en forme de tranches assez minces pour pouvoir supposer que la déformation et la contrainte ne varient pas dans l'épaisseur de chacune d'elles (Fig. 18). Des conditions aux limites adaptées au cas expérimental considéré sont

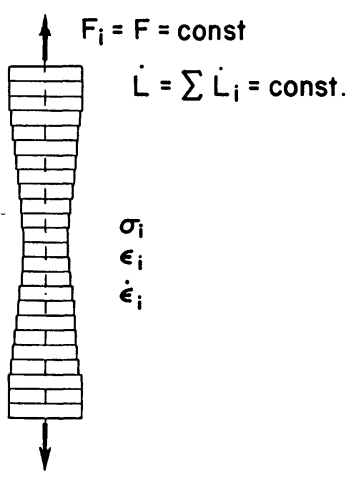

Fig. 18. - Représentation d'une éprouvette de traction discrétisée en tranches élémentaires pour une simulation par Différences Finies.

[Slice discretization of a tensile specimen for a Finite Difference simulation.] 
imposées aux éléments extrêmes de l'éprouvette (par exemple une vitesse de déplacement constante et un rayon constant). Ainsi, un état donné du système est caractérisé pour chaque élément $i \mathrm{du}$ modèle par des valeurs courantes de $\varepsilon_{i}, \dot{\varepsilon}_{i}, \sigma_{i}$ et $F_{\mathrm{T} i}$. Avec une discrétisation en 100 éléments, on obtient déjà une très bonne description des phénomènes à condition que les incréments de déformation de chaque élément soient assez petits.

A titre d'illustration, les profils et la courbe dynamométrique de la figure 19 montrent les résultats d'une simulation effectuée dans les conditions de l'expérience de la figure 10 . On constate que toutes les caractéristiques observées expérimentalement sont reproduites avec une grande fidélité par la simulation qui s'avère donc être un bon moyen pour prévoir les conditions d'instabilité plastique dans les cas pratiques. La méthode peut prendre en compte également les variations de $m$ avec $\varepsilon$ de même que les effets d'auto-échauffement (avec des hypothèses supplémentaires sur les conditions d'échange thermi-

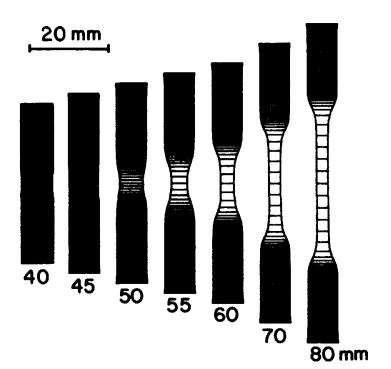

a)

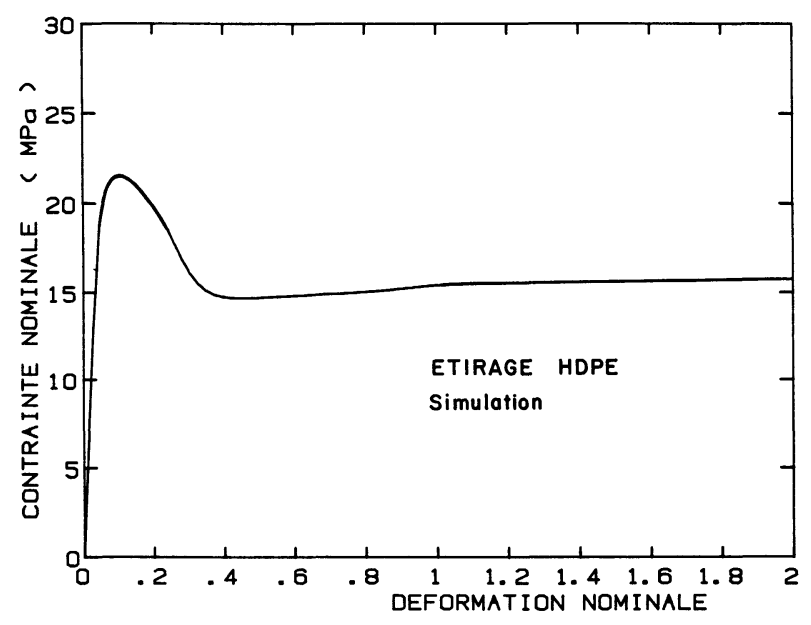

b)

Fig. 19. - Simulation par la Méthode des Différences Finies de l'évolution de la striction dans un échantillon cylindrique de polyéthylène à $22^{\circ} \mathrm{C}$ (cas expérimental de la Fig. 10).

[Finite Difference Simulation of necking in a cylindrical polyéthylène specimen at $22^{\circ} \mathrm{C}$ (experimental case in Fig. 10).]

\footnotetext{
REVUE DE PHYSIQUE APPLIQUÉE. - T. 23, N` 6, JUIN 1988
}

que par conduction et convection). Nous avons également étudié avec cette approche l'influence d'une prédéformation localisée ou uniforme sur la stabilité de la déformation [58].

4.4 Etirage DES Films MinCES. - Le cas de l'étirage de films minces en conditions de déformation plane a été également analysé par la Méthode des Différences Finies. Dans cette situation, la déformation transversale dans le plan du film est nulle. L'allongement du film est donc assumé seulement par la réduction d'épaisseur : $\varepsilon=\ln \left(e_{0} / e\right)$. D'après un calcul classique, la déformation effective s'écrit $\varepsilon_{\text {eff }}=2 / \sqrt{3}$. $\varepsilon$ et la contrainte effective $\sigma_{\text {eff }}=\sqrt{3} / 2 . \sigma$. Si une striction apparaît dans le

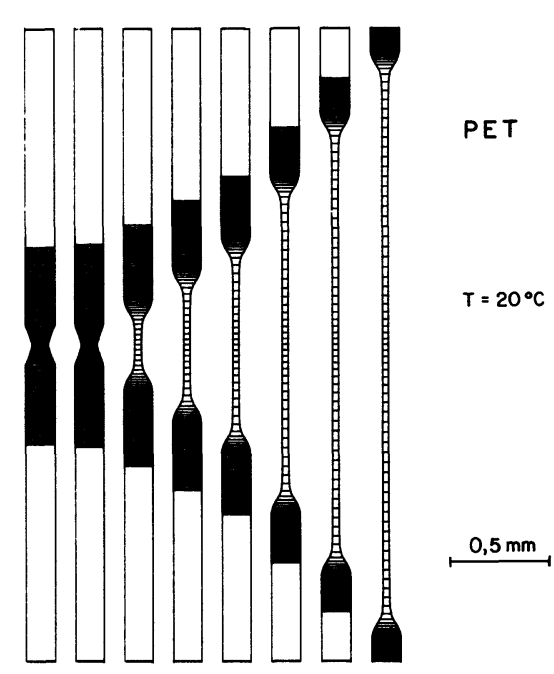

a)

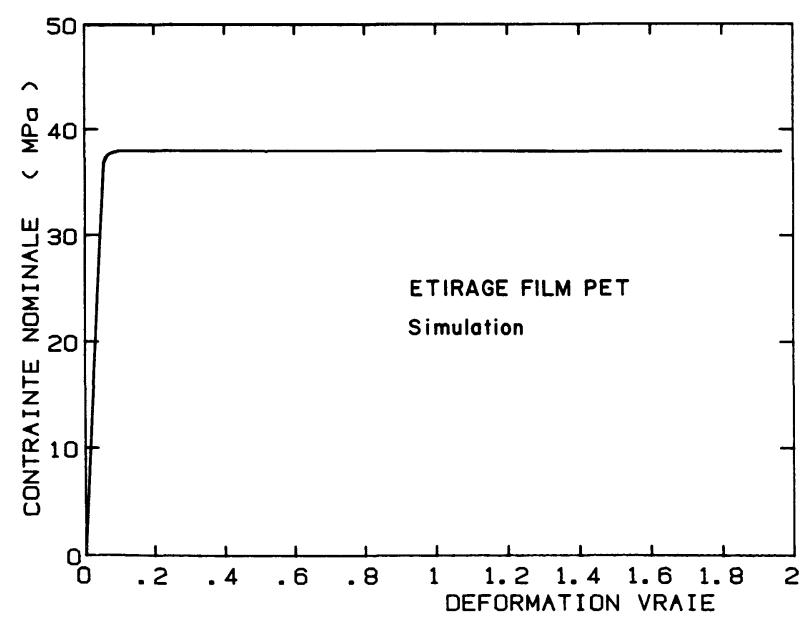

b)

Fig. 20. - Modélisation par la Méthode des Différences Finies de l'étirage d'un film mince de PET en déformation plane à $20^{\circ} \mathrm{C}$ (cas expérimental de la Fig. 11).

[Finite Difference simulation of necking in a thin film of PET in plane strain tension at $20^{\circ} \mathrm{C}$ (experimental case in Fig. 11).] 
film, il faut tenir compte en outre de l'effet de la courbure du profil d'épaisseur qui engendre une composante de contrainte transversale normale au plan du film (positive ou négative selon le sens de la courbure). La contrainte effective s'écrit alors $\sigma_{\text {eff }}=F_{\mathrm{T}} \cdot \sqrt{3} / 2, \sigma$ où $F_{\mathrm{T}}$ est un facteur de triaxialité qui se calcule par une formule analogue à (3) à partir d'une analyse appropriée au cas du mode de déformation plane. Dans la situation expérimentale illustrée à la figure 11 pour l'étirage d'un film mince de PET amorphe à la température ambiante, la simulation par la Méthode des Différences Finies s'applique aussi facilement que dans le cas précédent et on obtient les profils simulés et la courbe dynamométrique représentés à la figure 20 .

\section{Conclusions.}

Les méthodes d'analyse présentées ci-dessus ont permis de mieux comprendre qualitativement quels paramètres jouent le plus grand rôle dans les phénomènes d'instabilité sous étirage des matériaux polymères. Quantitativement, elles permettent dans des cas géométriquement simples de prévoir avec assez de précision la cinétique de formation et de propagation des strictions diffuses. Pour approcher avec encore plus de finesse les résultats expérimentaux, deux voies pourraient êtr recherchées :

- Une prise en compte des effets d'anisotropie introduits par le développement d'une texture de déformation dans le polymère étiré [54].

- Une analyse plus précise des effets de triaxialité des contraintes aboutissant à une formulation du facteur $F_{\mathrm{T}}$ meilleure que celle déduite du calcul de Bridgman.

Toutefois, la méthode des Différences Finies présente des limitations intrinsèques dans la mesure où elle ne donne pas accès aux variations des contraintes et des déformations suivant les dimensions transversales de l'échantillon et ne prend en compte qu'imparfaitement la triaxialité des contraintes et des déformations. La Méthode des Eléments Finis est certainement en mesure de remplir ce rôle de façon plus performante. Elle a été utilisée avec succès pour la simulation du développement de bandes de déformations mésoscopiques et de la striction macroscopique dans le cas d'éprouvettes métalliques [59]. Son application au cas des polymères est en progrès dans quelques centres de calcul spécialisés. Toutefois, la lourdeur des moyens informatiques et le coût des calculs qu'elle entraîne pour l'étude des instabilités d'étirage dans les matériaux visco-élasto-plastiques la limite encore aujourd'hui à des applications exemplaires. Les théories analytiques et les méthodes de simulations approchées, comme celles que nous avons présentées dans ce travail, demeurent très utiles pour rendre compte des phénomènes de base et modéliser systématiquement les cas réels.

\section{Bibliographie}

[1] Backofen, W. A., Deformation Processing (Addison-Wesley) Reading, Mass (1972).

[2] Semiatin, S. L. et Jonas, J. J., Formability and Workability of Metals ASM Ser. Metal Processing, Ed. H. L. Gegel (A.S.M.) Metals Park, Ohio (1984).

[3] Coffman, P. M., Plast. Eng. 8 (1977) 19.

[4] De Vries, A. J., Bonnebat, C. et Beautemps, J., J. Polym. Sci. Polym. Symposia 58 (1977) 109.

[5] Marquez-Lucero, A., Etude des Instabilités d'Etirage dans des Films Minces de Poly(Terephtalate d'Ethylene Glycol), Thèse de Docteur-Ingénieur, INPL, Nancy (1986).

[6] Kulkarni, K. M., Polym. Eng. Sci. 19 (1979) 474.

[7] Weynant, E. et Haudin, J. M., Matér. Tech. 3 (1982) 215.

[8] Parsons, B and WARd, I. M., Plast. Rubb. Processing Appl. 2 (1982) 215.

[9] Chretien, G., Matériaux Composites à matrice organique Technique et Documentation (1986) p. 429.

[10] Wilchinsky, Z. W., S.P.E. J. 3 (1966) 46.

[11] Warshavski, M. et ToKITA, N., S.P.E. 8 (1970) 55.
[12] G'SELL, Plastic Deformation of Amorphous and Semi-Crystalline Materials Eds. B. Escaig and C. G'Sell (Les Editions de Physique) Les Ulis (1982) p. 375.

[13] Mark, J. E., Physical Properties of Polymers (American Chemical Society) Washington D.C. (1984).

[14] Treloar, L. R. G., Rep. Prog. Phys. 36 (1973) 755.

[15] Schultz, J., Polymer Materials Science (PrenticeHall, Englewood Cliffs, N.J.) 1974.

[16] Haudin, J. M., Plastic Deformation of Amorphous and Semi-Crystalline Materials Op. Cit. p. 291.

[17] Yannas, I. V. et LuISE, R. R., The Strength and Stiffness of Polymers Eds. A. E. Zachariades et R. S. Porter (Marcel Dekker, New-York) 1983, p. 255.

[18] Kramer, E. J., Plastic Deformation of Amorphous and Semi-Crystalline Materials Op. Cit. p. 391.

[19] Escaig, B., Ann. Phys. 3 (1978) 207.

[20] Argon, A. S., Polymeric Materials (A.S.M.) Metals Park, Ohio (1975) p. 411.

[21] Bowden, P. B., et Raha, S., Phil. Mag. 29 (1974) 149. 
[22] G'SELL, C., The Strength of Metals and Alloys Ed. H. J. McQueen et al. (Pergamon Press) Oxford (1986) p. 1943.

[23] Li, J. C. M. et WU, J. B. C., J. Mater. Sci. 11 (1976) 445.

[24] Bridgman, P. W., Trans. Am. Soc. Met. 32 (1944) 553.

[25] G'Sell, C. et Jonas, J. J., J. Mater. Sci. 14 (1979) 583.

[26] G'Sell, C. et Jonas, J. J., J. Mater. Sci. 16 (1981) 1956.

[27] Jonas, J. J., Holt, R. A. et Coleman, C. E., Acta Metall. 24 (1976) 911.

[28] Hollomon, J. H., Trans. A.I.M.E. 162 (1945) 268.

[29] Hirth, J. P. et Lothe, J., Theory of Dislocations (McGraw-Hill) (1968).

[30] Weynant, E., Haudin, J. M. et G'Sell, C., J. Mat. Sci. 15 (1980) 2677.

[31] Hay, I. L. et Keller, A., Kolloid 2204 (1965) 43.

[32] Bauwens, J. C., J. Polym. Sci. A2 8 (1970) 893.

[33] Ender, D. H. et Andrews, E. D., J. Appl. Phys. 36 (1965) 3057.

[34] Kastelic, J. WR. et BAer, E., J. Macromol. Sci. (Phys) B 7 (1973) 679.

[35] Walker, N., Polymer 21 (1980) 857.

[36] Ishikawa, M., Narisawa, I. et Ogawa, H., Polymer J. 8 (1976) 391.

[37] Bauwens, J. C., Plastic Deformation of Amorphous and Semi-Crystalline Materials Op. Cit. p. 175.

[38] WARD, I. M., J. Mater. Sci. 6 (1971) 1397.

[39] Bowden, P. B. et Jukes, J. A., J. Mater. Sci. 7 (1972) 52.

[40] G'Sell, C. Boni, S. et Shrivastava, S., J. Mater. Sci. 18 (1983) 903.
[41] G'Sell, C. et Gopez, A. J., J. Mater. Sci. 18 (1985) 3462.

[42] Hill, R., J. Mech. Phys. Solids 1 (1952) 19.

[43] Hill, R. et Hutchinson, J. W., Ibid. 23 (1975) 239.

[44] Storen, S. et Rice, J. R., Ibid. 23 (1975) 421.

[45] Hutchinson, J. W. et NeAle, K. W., Ibid. 31 (1983) 405.

[46] Cross, A. et Haward, R. N., J. Polym. Sci. 11 (1973) 2423.

[47] Argon, A. S., The Inhomogeneity of Plastic Deformation (A.S.M.) Metals Park, Ohio (1973) p. 161.

[48] Bowden, P. B., Phil. Mag. 22 (1970) 455.

[49] Bowden, P. B. et RAHA, S., Ibid. 22 (1970) 463.

[50] Mills, N. J., Brit. Pol. J. 10 (1978) 1.

[51] G'Sell, C., Aly-Helal, N. A. et Jonas, J. J., J. Mater. Sci. 18 (1983) 1731.

[52] G'Sell, C., Marquez-Lucero, A., Gilormini, P. et Jonas, J. J., Acta Metall. 33 (1985) 759.

[53] G'Sell, C., Marquez-Lucero, A., Souahi, A. et Tong, Y. S., Instabilités Plastiques (Presses des Ponts-et-Chaussées) Paris (1985) p. 159.

[54] Cotto, D., Montheillet, F. J. et Haudin, J. M., C.R. Acad. Sc. Paris, 298 (1984) 511.

[55] Vincent, P. I., Polymer 1 (1960) 7.

[56] PAE, K. D., J. Mater. Sci. 12 (1977) 1209.

[57] Semiatin, S. L., Ghosh, A. K. et Jonas, J. J., Metall. Trans. 16A (1985) 2291.

[58] G'Sell, C. et Aly-Helal, A. N., Deformation, Yield and Fracture of Polymers Ed. the Plastic and Rubber Institute, London (1985) pp. 52-51.

[59] Pierce, D., Asaro, R. J. et Needleman, A., Acta Metall. 31 (1983) 1951. 